\title{
Recurrence rate of intramucosal gastric cancer with positive vertical margin due to lesion damage during endoscopic submucosal dissection
}

\author{
J. Hayasaka ${ }^{1,2}$, D. Kikuchi ${ }^{1}$, K. Nomura ${ }^{1}$, H. Odagiri ${ }^{1}$, Y. Ochiai ${ }^{1}$, Y. Suzuki ${ }^{1}$, Y. Fukuma ${ }^{1}$, M. Tanaka ${ }^{1}$, S. Yamashita $^{1}$, A. \\ Matsui $^{1}$, N. Inoshita ${ }^{1}$, M. Kitagawa ${ }^{2}$, S. Hoteya ${ }^{1}$ \\ (1) Department of Gastroenterology, Toranomon Hospital, 2-2-2, Toranomon, Minato-ku, Tokyo, Japan. ; (2) Department of Comprehensive Pathology, Graduate School \\ of Medical and Dental Sciences, Tokyo Medical and Dental University, Bunkyo-ku, Tokyo, Japan..
}

\begin{abstract}
Background and study aim : In principle, additional surgery is performed after endoscopic submucosal dissection for early gastric cancer if the vertical margin is positive, regardless of lesion damage. The recurrence rate of vertical margin-positive lesions due to lesion damage after endoscopic submucosal dissection is unknown, and unnecessary surgeries may be performed. In this study, we investigated whether there was a difference in the recurrence rate between vertical margin-positive lesions due to lesion damage and vertical margin-negative lesions.

Patients and methods : We included 1,294 intramucosal gastric cancer lesions that were resected by endoscopic submucosal dissection between January 2008 and December 2016, without additional surgery. The lesions were divided into the Damage and No damage groups based on vertical margin status. The Damage group had only one non-curative indication: a positive vertical margin due to lesion damage. The No damage group had no noncurative indications. We compared the recurrence rate between the Damage and No damage groups.

Results : The recurrence rates of the Damage and No damage groups were $0 \%(0 / 23 ; 95 \%$ confidence interval: $0-14.8 \%)$ and $0 \%$ $(0 / 1,271 ; 95 \%$ confidence interval: $0-0.003 \%)$, respectively, with no statistically significant difference.

Conclusions : In intramucosal gastric cancer, the recurrence rate of vertical margin-positive lesions due to lesion damage was $0 \%$, which did not differ from that of vertical margin-negative lesions with curative resection. Follow-up, instead of additional surgery, may be an option for patients with non-curative resection when the only non-curative indication is a positive vertical margin due to lesion damage. (Acta gastroenterol. belg., 2021, 84, 289-294).
\end{abstract}

Key words : early gastric cancer, endoscopic submucosal dissection, neoplasm, recurrence, vertical margin.

\section{Introduction}

Compared with surgery, endoscopic submucosal dissection (ESD) is widely used as a less invasive treatment for early gastric cancer (EGC), with an equivalent lymph node metastasis rate after surgery. According to the Japanese guidelines (1-4) for gastric cancer, based on past reports (5-8), certain criteria must be fulfilled for resection to be determined as curative; otherwise, it is considered non-curative. If a positive horizontal margin (HM) or piecemeal resection is the only indication for non-curative resection, the lesion is considered as curability $\mathrm{C}-1$. A lesion with other noncurative indications, including a positive vertical margin (VM), is considered as curability C-2. Lesions with curability $\mathrm{C}-1$ or $\mathrm{C}-2$ are considered to need additional treatment. Given that the risk of recurrence after ESD with curability C-1 for EGC is low $(9,10)$, according to the Japanese guidelines, repeated (secondary) ESD, close observation, and endoscopic coagulation may be considered as additional treatments, instead of surgical resection (1-4,11-16). After ESD with curability C-2 for EGC, an additional surgical resection is the standard treatment option (1-4). However, only $5-10 \%$ of cases exhibit lymph node metastases after additional surgery (17-22), and additional surgical resection may be unnecessary for most patients. In fact, cancer-specific survival was as high as $97.5 \%$ even without additional treatment after non-curative resection (23).

When lesions are damaged, it is difficult to evaluate the margins due to the burn effect, and the VM is often positive. When the VM is positive, we would principally consider additional surgery. However, in practice, we often choose merely to follow up with the patient, as we think that the risk of recurrence is low when the only noncurative indication is a positive VM due to lesion damage. However, the recurrence rate of such lesions is unknown. Moreover, it is unknown whether there is a difference in the recurrence rate between lesion with a positive VM due to being damaged and lesions with a negative VM. Therefore, in this retrospective study, we aimed to assess the recurrence rate of VM-positive lesions due to damage where the only non-curative indication was a positive VM, as well as to investigate whether there is a difference in the recurrence rate between such lesions and VM-negative lesions.

\section{Methods}

\section{Study design and population}

In total, 1,885 consecutive patients with 2,121 lesions who underwent ESD for EGC in Toranomon Hospital between January 2008 and December 2016 were included. Of these, 1,854 EGCs were intramucosal. Specimens with multiple lesions were excluded, as it may have

Correspondence to : Junnosuke Hayasaka, MD, Department of Gastroenterology, Toranomon Hospital, 2-2-2, Toranomon, Minato-ku, Tokyo, Japan. Phone : +8133588-1111, Fax : +813-3560-7878.

E-mail : bandwagondane@gmail.com

Submission date : 20/05/2020

Acceptance date : 13/09/2020 


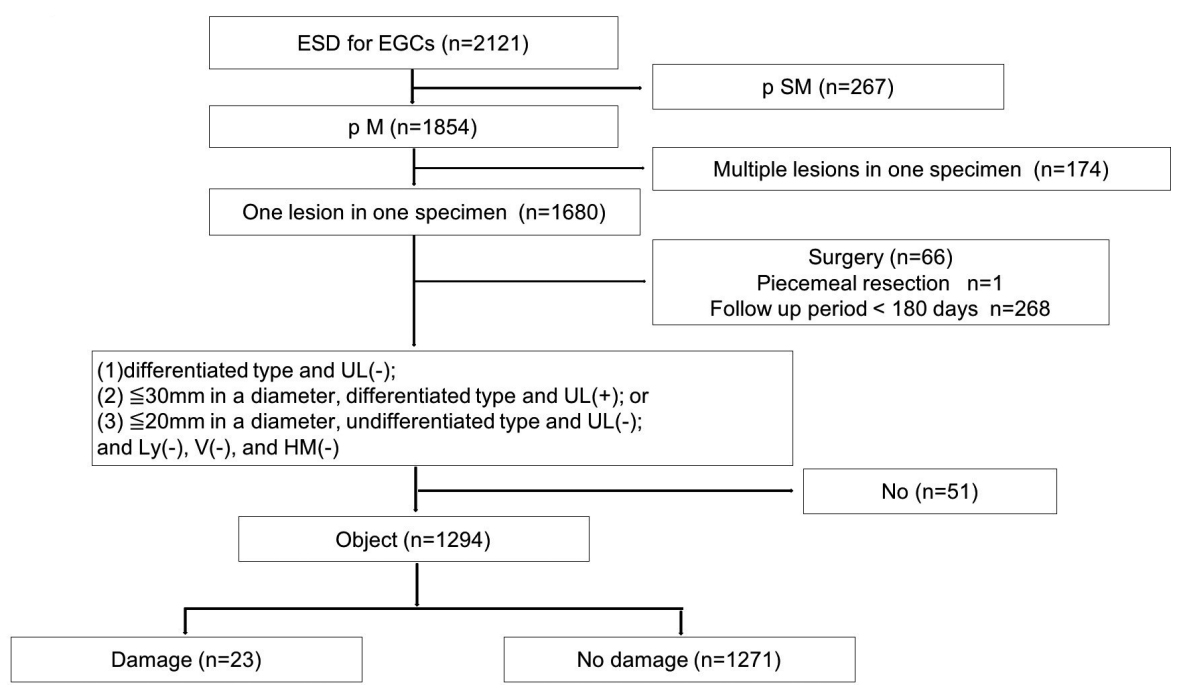

Figure 1 - Flowchart of the enrollment process. $p M$, pathologic intramucosal carcinoma. $p S M$, pathologic submucosal invasive carcinoma. $E S D$, endoscopic submucosal dissection. $E G C$, early gastric cancer. $U L$, ulcerative finding. $L y$, lymphatic invasion. $V$, vascular invasion. $H M$, horizontal margin.

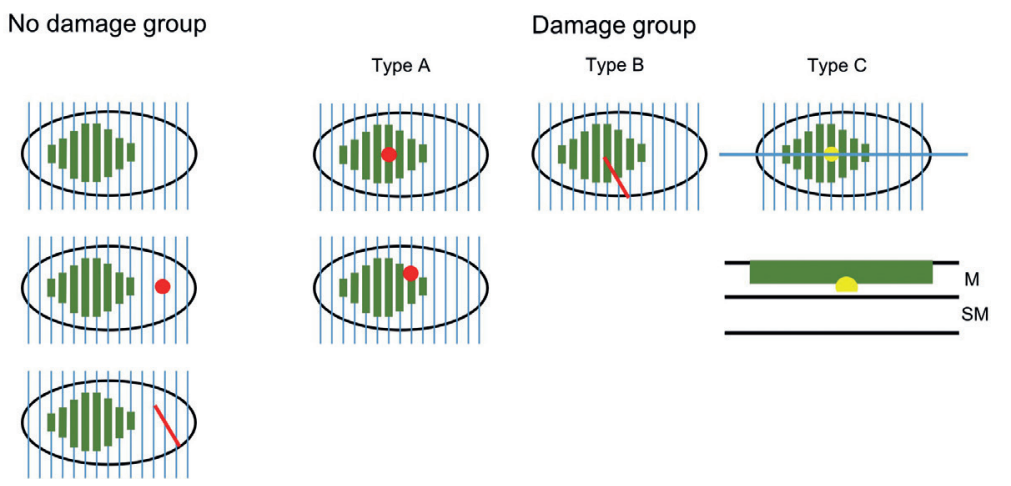

Figure 2 - Types of damage. The lesions are indicated by green lines. A red dot indicates a hole. A red line indicates a split. A yellow dot indicates burn damage without a hole. The No damage group includes damage outside the lesion. The Damage group was divided into three types. Type $A$ consisted of a hole in the lesion. It included a hole that occupied the horizontal margin. Type B consisted of a split in the lesion. Type $C$ consisted of crushed and/or burned tissue with no hole or split, where the VM was difficult to evaluate. As a specific example, a cut surface is presented. $M$, mucosal layer. $S M$, submucosal layer.

confounded the cause of recurrence. Of the remaining 1,680 lesions, 335 were excluded because of surgery, piecemeal resection, or a follow-up period of $<180$ days. Ultimately, 1,294 lesions satisfied the inclusion criteria of no lymphatic invasion, no vascular invasion, and a negative HM, and one of the following: (1) differentiated type and no ulcerative finding (UL); (2) tumor size $\leq 30$ $\mathrm{mm}$, differentiated type, and UL; or (3) tumor size $\leq 20$ $\mathrm{mm}$, undifferentiated type, and no UL (Figure 1).

The lesions were classified, endoscopically or pathologically, into the Damage and No damage groups. Lesions in the Damage groups had a positive VM as the only non-curative indication, while the No damage groups had no non-curative indications.

Indications for additional surgical resection were determined according to the current Japanese gastric cancer guidelines (1-4). Additional surgical resection or follow-up was decided based on patients' general condition, background, and preference.

\section{Ethical statements}

All patients provided written informed consent to undergo ESD. This study was conducted in accordance with the Declaration of Helsinki. Moreover, this study was approved by the Ethics Committee of Toranomon Hospital (approval number: 1827).

\section{Definition of lesion damage}

Lesion damage was defined as crush or burn damage, caused by the knife during resection, that affected the VM. We included both accidental damage and damage due to a shallow resection layer. Lesion damage was divided into three types (Figure 2). Type A (hole type) damage consisted of a hole in the lesion. As sections of these lesions were lost, the VM could not be evaluated. Type B (split type) damage consisted of a split in the lesion. The split sections were often accompanied with denaturation 


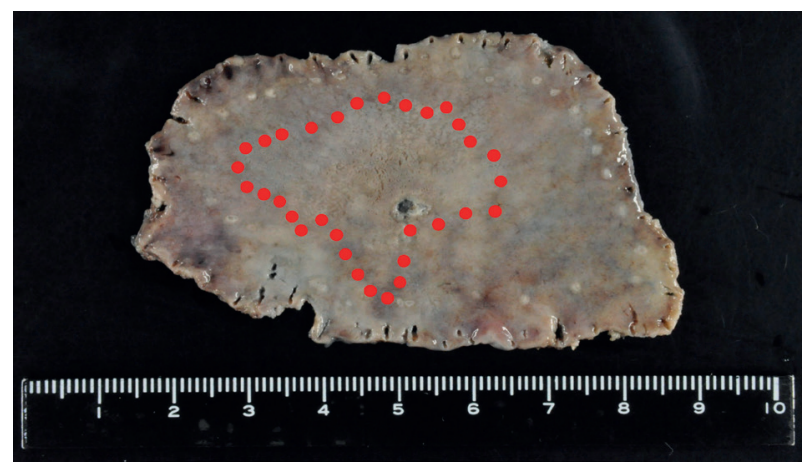

Figure 3 - Type A lesion damage. The lesion was surrounded by red points and had a hole. The hole made it difficult to evaluate the vertical margin, and the results showed that the vertical margin was positive.

due to burning, which made it difficult to evaluate VM. Type C (crush/burn type) damage consisted of crushed and/or burned tissue with no hole or split, where the VM was difficult to evaluate, especially where the damage extended to the VM. A representative case of Type A is presented in Figure 3.

\section{Main outcome measures}

We compared the recurrence rates and clinicopathological characteristics between the Damage and No Damage groups.

\section{ESD procedure}

The ESD procedure was performed with a dual knife (KD-650Q ; Olympus, Tokyo, Japan), flex knife (KD630L ; Olympus), or hook knife (KD-620LR ; Olympus) through a single-channel endoscope (GIF-Q260J ; Olympus) or a 2-channel endoscope (2TQ260M ; Olympus). The procedure was performed with an electrosurgical generator (ICC 200 or VIO 300D, Erbe, Tubingen, Germany). Marking dots were placed at least 5 $\mathrm{mm}$ away from the demarcation line. After a submucosal injection of a glycerol solution $(10 \%$ glycerol and $5 \%$ fructose; Chugai Pharmaceutical, Tokyo, Japan) containing indigo carmine, a mucosal incision was made outside the marker dots. Sodium hyaluronate (MucoUp ${ }^{\circledR}$; Johnson \& Johnson, NJ, USA) was used for the submucosal injection where the mucosal elevation was insufficient.

After the mucosal incision was made, the submucosal layer was dissected, and resection was performed. Bleeding during ESD was coagulated with a knife or a pair of hemostatic forceps (HDB2422W ; Pentax, Tokyo, Japan). Visible vessels on the artificial ulcer after ESD were prophylactically coagulated with hemostatic forceps or clipped with EZ Clips (HX-610-090/HX-610090S/HX-610-135 ; Olympus).

\section{Pathological evaluation}

The specimens were fixed with formalin and cut into 2-mm slices. Histopathological diagnosis was determined according to the Japanese Classification of Gastric Carcinoma (24). Positive margins in this study were defined as cases in which the margins were difficult to evaluate or cases in which tumor tissue remained at the margins.

Although the exact depth of lesions with positive margins were unknown, if the section that could be evaluated pathologically was only intramucosal, the lesion was diagnosed as intramucosal cancer.

\section{Follow-up}

Principally, a second endoscopic follow-up was performed at 8 weeks after ESD. Thereafter, endoscopic follow-ups were performed in 6- or 12-month intervals. Computed tomography or abdominal ultrasonography was performed at the discretion of the attending physician. Recurrence, including local and metastatic, was evaluated pathologically or radiologically. Metastatic recurrence included that of lymph nodes or other organs.

\section{Statistical analysis}

Continuous, non-normally distributed variables are expressed as the mean and interquartile range. Mean quantitative values were compared using the Wilcoxon rank-sum test. Categorical variables were compared using the Fisher exact test.

Statistical significance was set at $\mathrm{P}<0.05$. All analyses were performed with $\mathrm{R}$ software version 3.1.2 (The R Foundation for Statistical Computing, Vienna, Austria).

\section{Results}

The Damage group included 23 lesions, whereas the No damage group comprised 1,271 lesions. All VMpositive lesions were included in the Damage group by definition, as they were restricted to cases of intramucosal cancer. Of the 23 lesions in the Damage group, 4 were VM-positive lesions and 19 were lesions for which it was difficult to evaluate the margin.

The clinical characteristics of the lesions in the two groups are summarized in Table 1. Damage patterns were Types A, B, and C in 16 (69.6\%), 4 (17.4\%), and 7 lesions $(30.4 \%)$, respectively. The recurrence rates were $0 \%$ (0 lesions) (95\% confidence interval [CI]: $0-14.8)$ in the Damage group and $0 \%$ (0 lesions) (95\% CI: $0-0.003$ ) in the No damage group. The median follow-up period was 945 days (interquartile range [IQR]: 760-2,293) in the Damage group and 1,477 days (IQR: $964-2,380$ ) in the No damage group. There was no difference in the recurrence rate and follow-up period between the two groups. ULs were statistically significantly more frequent in the Damage group than in the No damage group. 
Table 1. - Characteristics of lesions

\begin{tabular}{|c|c|c|c|}
\hline & Damage $(n=23)$ & No damage $(\mathrm{n}=1271)$ & $P$ value \\
\hline \multicolumn{4}{|l|}{ Damage pattern } \\
\hline Type A & $16(69.6 \%)$ & $0(0 \%)$ & \\
\hline Type B & $4(17.4 \%)$ & $0(0 \%)$ & \\
\hline Type C & $7(30.4 \%)$ & $0(0 \%)$ & \\
\hline Age (years), median (IQR ) & $75.0(68.0-80.0)$ & $71.0(64.0-77.0)$ & 0.057 \\
\hline Sex & & & $>0.99$ \\
\hline Male & $19(82.6 \%)$ & $1016(79.9 \%)$ & \\
\hline Female & $4(17.4 \%)$ & $255(20.1 \%)$ & \\
\hline Location & & & 0.118 \\
\hline U^ & $6(30.0 \%)$ & $191(16.0 \%)$ & \\
\hline $\mathrm{M} \bullet \uparrow+\mathrm{L} \bullet \bullet \bullet \uparrow$ & $14(70.0 \%)$ & $1005(84.0 \%)$ & \\
\hline Postoperative stomach & $3(13.0 \%)$ & $75(5.9 \%)$ & 0.157 \\
\hline Macroscopic type & & & 0.34 \\
\hline $0-\mathrm{I}$ & $0(0 \%)$ & $35(2.8 \%)$ & \\
\hline 0-IIa & $9(39.1 \%)$ & $374(29.4 \%)$ & \\
\hline $0-\mathrm{IIb}$ & $2(8.7 \%)$ & $56(4.4 \%)$ & \\
\hline 0 -IIc & $12(52.2 \%)$ & $806(63.4 \%)$ & \\
\hline Specimen size (mm), median (IQR) & $44.0(38.5-60.0)$ & $37.0(30.0-46.0)$ & 0.005 \\
\hline Tumor size (mm), median (IQR) & $20.0(12.5-27.5)$ & $13.0(9.0-20.0)$ & 0.014 \\
\hline Histological type & & & 0.58 \\
\hline Differentiated & $22(95.7 \%)$ & $1,225(96.4 \%)$ & \\
\hline Undifferentiated & $1(4.3 \%)$ & $46(3.6 \%)$ & \\
\hline Ulcerative findings & & & 0.001 \\
\hline Absent & $15(65.2 \%)$ & $1155(90.9 \%)$ & \\
\hline Present & $8(34.8 \%)$ & $116(9.1 \%)$ & \\
\hline Vertical margin & & & $<0.001$ \\
\hline Negative & $0(0 \%)$ & $1269(100 \%)$ & \\
\hline Positive & $35(100 \%)$ & $0(0 \%)$ & \\
\hline \multirow{2}{*}{ Recurrence } & $0(0 \%)$ & $0(0 \%)$ & \multirow{2}{*}{$>0.99$} \\
\hline & [95\% CI ৬৬৬४४:0-14.8] & [95\% CI:0-0.003] & \\
\hline Follow-up period (days), Median (IQR) & $945(760-2,293)$ & $1,477(964-2,380)$ & 0.24 \\
\hline
\end{tabular}

$\bullet:$ Upper third of the stomach, : middle third of the stomach, : lower third of the stomach, interval.

Specimen and tumor size were statistically significantly larger in the Damage group than in the No damage group.

\section{Discussion}

In the present study, the recurrence rate of intramucosal gastric cancer lesions with a positive-VM due to lesion damage was $0 \%$, and there was no difference in the recurrence rate between such lesions and VM-negative lesions. Therefore, lesions for which the only non-curative indication is a positive VM can be treated similarly to lesions that have been curatively resected: follow-up may be sufficient. One strength of this study is that it excluded lesions with submucosal invasion and multiple lesions, making it possible to evaluate the recurrence rate of VMpositive lesions. To our knowledge, this is the first study to evaluate the recurrence rate of VM-positive lesions due to lesion damage after ESD in intramucosal gastric cancer.

In the present study, the recurrence rate of such lesions in intramucosal gastric cancer was $0 \%$, and there was no difference in the recurrence rate between those lesions and VM-negative lesions. Tumor size, lymphatic invasion, venous invasion, histological type, UL, and margins have all been demonstrated to play a role in recurrence after $\operatorname{ESD}(5,6,20,23,25-28)$. Among those, a positive VM is involved in lymph node metastasis $(9,10)$. However, local recurrence and distant metastasis have not been observed when the tumor depth was considered mucosal or submucosal $\leq 500 \mu \mathrm{m}$, even with a positive VM (28). Moreover, in a large multicenter retrospective study, Hatta et al. (23) reported that a positive VM was a significant predictive factor of recurrence of lymph node metastasis upon univariate analysis, but that only lymphatic invasion was an independent predictive factor of recurrence of lymph node metastasis upon multivariate analysis. Therefore, a positive VM in intramucosal gastric cancer has a low recurrence potential, which may be the reason for the absence of recurrence in our patients with positive VM due to lesion damage.

In the Damage group, ULs were statistically significantly more frequent, and tumor size was statistically significantly larger. Therefore, the risk of recurrence was not lower in the Damage group than in the No damage group. Other possible reasons for non-recurrence could be the misdiagnosis of positive VM due to crush or burn 
damage. In a previous study, the local/distant recurrence rate was $0 \%$ when crush or burn damage was the cause of a positive HM (10), possibly due to misdiagnosis. Similarly, in the present study, lesions in the Damage group may have been misdiagnosed as VM-positive due to crush or burn damage, resulting in $0 \%$ recurrence. In fact, only 4 of 23 lesions classified as VM-positive had identifiable tumors remaining in the VM. Actually, in more than half of patients who undergo additional surgical resection after a positive VM, no residual tumors are observed $(25,28-30)$. Therefore, follow-up, instead of additional surgery, can be considered in the case of lesions classified as VM-positive due to lesion damage in intramucosal gastric cancer after ESD.

In this study, there were several differences in the characteristics between the Damage and No damage groups. Although we have not considered the predictive factors of positive VM in this study, we speculate that the differences in specimen size and UL may be such predictive factors, as they make it difficult to perform ESD.

In this study, the recurrence rate of VM-positive lesions due to lesion damage after ESD did not differ from that of VM-negative lesions, when evaluating only intramucosal cancer. Although the true depths of the lesions were unknown, the results suggest that VMpositive lesions can be treated similarly to VM-negative lesions, when the non-curative indication is only a positive VM. Therefore, for intramucosal gastric cancer, additional surgeries may not be necessary after ESD when the only non-curative indication is a positive VM due to lesion damage, and follow-ups may be sufficient. Of course, the priority should be to dissect specimens without causing lesion damage.

There were certain limitations to this study. First, this was a single-center retrospective study based on medical records. However, ethical considerations prevent the prospective collection of VM-positive lesions, as they are undesirable. Second, the sample of VM-positive lesions was small. Lastly, since the median follow-up period was only 3-4 years, we might have underestimated the recurrence rate. However, it has been reported that many recurrences after ESD for EGC occur within 3 years $(21,31,32)$. Even in a prospective study with a 15 year follow-up period after surgical treatment of gastric cancer, most cases of recurrence occurred within 2-2.5 years (33). Moreover, Yamada et al. (34) reported that, upon univariate and multivariate analysis, a positive VM was not a risk factor for recurrence $>2$ years without additional surgery after ESD with curability C-2. Therefore, we consider it likely that a positive VM will likely have little impact on long-term recurrence after ESD with curability C-2. Thus, we believe that, although a longer observation period is preferred, our follow-up period is acceptable. A multi-center, retrospective study with a long observation period is required in future to verify results from this study.
In conclusion, in intramucosal gastric cancer, the recurrence rate of VM-positive lesions due to lesion damage after ESD was $0 \%$, which was not different from that of VM-negative lesions with curative resection. It may be sufficient to follow up when the only non-curative indication is a lesion classified as VM-positive due to lesion damage in intramucosal gastric cancer, rather than to perform additional surgery.

\section{Ethical approval}

All procedures performed in studies involving human participants were in accordance with the ethical standards of the institutional research committee and with the 1964 Helsinki declaration and its later amendments or comparable ethical standards.

\section{Informed consent}

For this type of study, formal consent was not required.

\section{Conflict of interest}

The authors declare that they have no conflict of interest for this article.

\section{Funding}

None.

\section{References}

1. JAPANESE GASTRIC CANCER ASSOCIATION. Japanese gastric cancer treatment guidelines 2014 (ver. 4). Gastric Cancer, 2017, 20 : 1-19.

2. ONO H., YAO K., FUJISHIRO M., ODA I., NIMURA S., YAHAGE N., et al. Guidelines for endoscopic submucosal dissection and endoscopic mucosal resection for early gastric cancer. Dig. Endosc., 2016, 28 : 3-15.

3. JAPANESE GASTRIC CANCER ASSOCIATION. Gastric cancer treatment guideline. 5th ed. Tokyo: Kaneharasyuppan, 2018. [in Japanese].

4. ONO H., YAO K., FUJISHIRO M., ODA I., UEDO F., NIMURA S., et al. Guidelines for endoscopic submucosal dissection and endoscopic mucosal resection for early gastric cancer (ver. 2). Gastroenterological. Endoscopy, 2020, $62:$ 273-290. [in Japanese].

5. GOTODA T., YANAGISAWA A., SASAKO M., ONO H., NAKANISHI Y., SHIMODA T., et al. Incidence of lymph node metastasis from early gastric cancer: estimation with a large number of cases at two large centers. Gastric Cancer, 2000, $3: 219-225$

6. HIRASAWA T., GOTODA T., MIYATA S., KATO Y., SHIMODA T., TANIGUCHI H., et al. Incidence of lymph node metastasis and the feasibility of endoscopic resection for undifferentiated-type early gastric cancer. Gastric Cancer, 2009, $12:$ 148-152.

7. HASUIKE N., ONO H., BOKU N., MIZUSAWA J., TAKIZAWA K., FUKUDA H., et al. A non-randomized confirmatory trial of an expanded indication for endoscopic submucosal dissection for intestinal-type gastric cancer (cT1a) : the Japan Clinical Oncology Group study (JCOG0607). Gastric Cancer., 2018, 21 : 114-123.

8. TAKIZAWA K., KAWATA N., TANAKA M., KAKUSHIMA N., IMAI K., MATSUBAYASHI H., et al. Clinicopathological characteristics of mixed histological type intramucosal gastric cancers with different patterns. Stom. Intest., 2013, 48 : 1567-1579. [in Japanese].

9. SUZUKI H., ODA I., ABE S., SEKIGUCHI M., NONAKA S., YOSINAGA S., et al. Clinical outcome of early gastric cancer patients after noncurative endoscopic submucosal dissection in a large consecutive patients series. Gastric cancer, 2017, 20 : 679-689.

10. NUMATA N., OKA S., TANAKA S., KAGEMOTO K., SANOMURA Y., YOSHIDA S., et al. Risk factors and management of positive margin in early gastric cancer resected by en bloc endoscopic submucosal dissection. Gastric cancer, 2015, $18: 332-338$. 
11. HIGASHIYAMA M., OKA S., TANAKA S., SANOMURA Y., YOSHIDA S., HIYAMA T., et al. Outcome of endoscopic submucosal dissection for gastric epithelial neoplasm in relationship to endoscopic classifications of submucosal fibrosis. Gastric Cancer, 2013, 16 : 404-410.

12. SEKIGUCHI M., SUZUKI H., ODA I., ABE S., NONAKA S., YOSHINAGA S., et al. Risk of recurrent gastric cancer after endoscopic resection with a positive lateral margin. Endoscopy, 2014, 46 : 273-278.

13. YOON H., KIM SG., CHOI J., IM JP., KIM JS., KIM WH., et al. Risk factor of residual or recurrent tumor in patients with a tumor-positive resection margin after endoscopic resection of early gastric cancer. Surg. Endosc., 2013, $27: 1561-1568$

14. BAE SY., JANG TH., MIN BH., LEE JH., RHEE PL., RHEE JC., et al. Early additional endoscopic submucosal resection in patients with positive lateral resection margins after initial endoscopic submucosal dissection for early gastric cancer. Gastrointest. Endosc., 2012, 75 : 432-436.

15. HOTEYA S., IIZUKA T., KIKUCHI D., MITANI T., MATSUI A., OGAWA O., et al. Secondary endoscopic submucosal dissection for residual or recurrent tumors after gastric endoscopic submucosal dissection. Gastric Cancer, 2014, $17: 697-702$.

16. KIKUCHI D., IIZUKA T., HOTEYA S., YAMADA A., FURUHATA T., YAMASHITA S., et al. Safety and efficacy of secondary endoscopic submucosal dissection for residual gastric carcinoma after primary endoscopic submucosal dissection. Digestion, 2012, $86: 288-293$

17. RYU KW., CHOI IJ., DOH YW., KOOK MC., KIM CG., PARK HJ., et al. Surgical indication for non-curative endoscopic resection in early gastric cancer. Ann. Surg. Oncol., 2007, $14:$ 3428-3434.

18. ODA I., GOTODA T., SASAKO M., SANO T., KATAI H., FUKAGAWA T., et al. Treatment strategy after non-curative endoscopic resection of early gastric cancer. Br. J. Surg., 2008, 95 : 1495-1500.

19. ITO H., INOUE H., IKEDA H., ODAKA N., YOSHIDA A., SATODATE H., et al. Surgical outcomes and clinicopathological characteristics of patients who underwent potentially noncurative endoscopic resection for gastric cancer: a report of a single-center experience. Gastroenterol. Res. Pract., 2013, 2013 : 427405.

20. SON SY., PARK JY., RYU KW., EOM BW., YOON HM., CHO SJ., et al. The risk factors for lymph node metastasis in early gastric cancer patients who underwent endoscopic resection : is the minimal lymph node dissection applicable? A retrospective study. Surg. Endosc., 2013, 27 : 3247-3253.

21. YANG HJ., KIM SG., LIM JH., CHOI J., IM JP., KIM JS., et al. Predictors of lymph node metastasis in patients with non-curative endoscopic resection of early gastric cancer. Surg. Endosc., 2015, 29 : 1145-1155.

22. KIM ER., LEE H., MIN BH., LEE JH., RHEE PL., KIM JJ., et al. Effect of rescue surgery after non-curative endoscopic resection of early gastric cancer. Br. J. Surg., 2015, 102 : 1394-1401.
23. HATTA W., GOTODA T., OYAMA T., KAWATA N., TAKAHASHI A., YOSHIFUKU Y., et al. Is radical surgery necessary in all patients who do not meet the criteria for endoscopic submucosal dissection of early gastric cancer? A multicenter retrospective study in Japan. J. Gastroenterol., 2016, $52: 175-184$.

24. JAPANESE GASTRIC CANCER ASSOCIATION. Japanese classification of gastric carcinoma : 3rd English edition. Gastric Cancer, 2011, 14: 101-112.

25. NAGANO H., OHYAMA S., FUKUNAGA T., SETO Y., FUJISAKI J., YAMAGUCHI T., et al. Indications for gastrectomy after incomplete EMR for early gastric cancer. Gastric Cancer, 2005, 8 : 149-154.

26. HANAOKA N., TANABE S., MIKAMI T., OKAYASU I., SAIGENJI K. Mixed-histologic-type submucosal invasive gastric cancer as a risk factor for lymph node metastasis: feasibility of endoscopic submucosal dissection. Endoscopy., 2009, $41:$ 427-432.

27. FUJIMOTO A., ISHIKAWA Y., AKISHIMA-FUKASAWA Y., ITO K. AKASAKA Y., TAMAI S., et al. Significance of lymphatic invasion on regional lymph node metastasis in early gastric cancer using LYVE-1 immunohistochemical analysis. Am. J. Clin. Pathol., 2007, 127 : 82-88.

28. NOH GY., KU HR., KIM YJ., PARK SC., KIM J., HAN CJ., et al. Clinical outcomes of early gastric cancer with lymphovascular invasion or positive margin vertical resection margin after endoscopic submucosal dissection. Surg. Endosc., 2015, $29:$ 2583-2589.

29. LEE HJ., JANG YJ., KIM JH., PARK SS., PARK SH., PARK JJ., et al. Clinical outcomes of gastrectomy after incomplete EMR/ESD. J. Gastric Cancer, 2011, $11:$ 162-166.

30 JUNG H., BAE JM., CHOI MG., NOH JH., SOHN TS., KIM S. Surgical outcome after incomplete endoscopic submucosal dissection of gastric cancer. Br. J. Surg., 2011, 98 : 73-78.

31. SAKA M., KATAI H., FUKAGAWA T, NIJJAR R, SANO T. Recurrence in early gastric cancer with lymph node metastasis. Gastric Cancer, 2008, 11 : 214-218.

32. YOUN HG., AN JY., CHOI MG., NOH JH., SOHN TS., KIM S. Recurrence after curative resection of early gastric cancer. Ann. Surg. Oncol., 2010, 17 : 448-454.

33. SONGUN I., PUTTER H., KRANENBARG EM., SASAKO M., VAN DE VELDE CJ. Surgical treatment of gastric cancer: 15-year follow-up results of the randomised nationwide Dutch D1D2 trial. Lancet Oncol., 2010, 11 : 439-449.

34. YAMADA S., HATTA W., SHIMOSEGAWA T., TAKIZAWA K., OYAMA T., KAWATA N., et al. Different risk factors between early and late cancer recurrences in patients without additional surgery after noncurative endoscopic submucosal dissection for early gastric cancer. Gastrointest. Endosc., 2019, 89 : 950-960. 